\title{
Analytic Properties of a Class of Hyperboloidal Beams in Nearly-Spheroidal Fabry-Perot Optical Cavities
}

\author{
Vincenzo Galdi* \\ Giuseppe Castaldi* \\ Vincenzo Pierro* \\ Innocenzo M. Pinto* \\ Juri Agresti ${ }^{\dagger, \ddagger}$ \\ Riccardo DeSalvo
}

\begin{abstract}
This paper deals with a study of the analytic structure and properties of a family of "hyperboloidal" beams (HBs) which spans from the standard Gaussian-beam configuration to "flat-top" beam configurations of interest for low-thermalnoise optical cavities for gravitational interferometry. In this connection, starting from a GaussLaguerre-type representation, a generalized duality relation (involving fractional Fourier transform operators of complex order) between two generic HBs is derived.
\end{abstract}

\section{INTRODUCTION}

Optical beams exhibiting a nearly uniform ("flattop") intensity distribution at a certain transverse plane are encountered and/or desirable in several practical applications, including lasers with highgain, and Fabry-Perot optical cavities with mirrors featuring variable reflectivity or graded-phase profiles. The reader is referred to [1]-[5] and the references therein for an introduction to the subject and several models and application examples.

Recently, there has been a strong interest toward application of these beams to advanced interferometric gravitational-wave detectors, as a possible device for reducing the intrinsic thermal noise of the optical-cavity mirrors, which currently sets the limiting sensitivity. The underlying idea is that a flat-top (commonly referred to as "mesa") beam, as compared to the standard Gaussian beam (GB) profile currently in use, is capable of providing a better averaging over the beam profile of the thermally-induced surface fluctuations [6]. In this framework, within the advanced Laser Interferometer Gravitational-wave Observatory (LIGO) experiment [7], Fabry-Perot optical cavities with

\footnotetext{
*Waves Group, Department of Engineering, University of Sannio, Corso Garibaldi 107, I-82100 Benevento, Italy, e-mail: vgaldi@unisannio.it, castaldi@unisannio.it, pierro@unisannio.it, pinto@sa.infn.it, tel.: +390824 385809, fax: +390824305838.

${ }^{\dagger}$ Department of Physics, University of Pisa, Largo Pontecorvo 3, I-56127 Pisa, Italy, e-mail: agresti@df .unipi.it

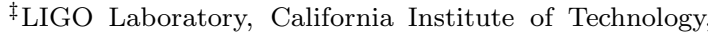
1200 California Blvd., Pasadena CA 91125, USA, e-mail: desalvo@ligo.caltech.edu, tel.: +1 626395 2968, fax: +1 6263049834 .
}

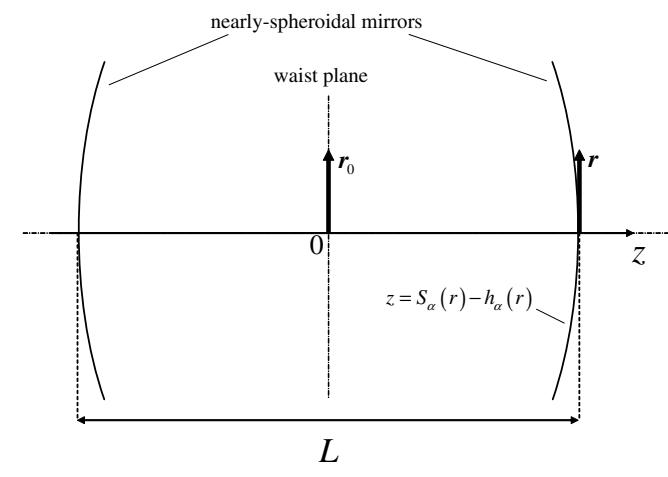

Figure 1: Problem schematic (see text).

non-spherical mirrors capable of supporting mesa beams are being actively investigated [8]. In order to reduce the severe tilt-instability problems affecting the originally conceived nearly-flat, "Mexicanhat-shaped" mirror configuration [9], a nearlyconcentric mirror configuration was proposed. In $[9,10]$, the nearly-flat (FM) and nearly-concentric mesa (CM) configurations were found to be connected through a Fourier-type duality relation, which allows a one-to-one mapping between all the corresponding eigenmodes. In [11], a further generalization was derived, in terms of a one-parameter family of "hyperboloidal" beams (HBs) supported by nearly-spheroidal mirrors, which allows continuous spanning from the FM to the CM configurations, including the standard GB case.

In this paper, we investigate the analytic properties of generic HBs, through the derivation of a Gauss-Laguerre (GL) representation and the extension of the Fourier-type duality relations in $[9,10]$.

\section{HYPERBOLOIDAL BEAMS}

\subsection{Background}

We refer to the problem geometry illustrated in Fig. 1, with a perfectly symmetric Fabry-Perot optical cavity composed of two nearly-spheroidal mirrors separated by a distance $L$ along the $z$-axis of a 
Cartesian $(x, y, z)$ coordinate system. The transverse coordinates at the waist $(z=0)$ and mirror $(z=L / 2)$ planes are denoted by $\mathbf{r}_{0} \equiv x_{0} \hat{\mathbf{x}}+y_{0} \hat{\mathbf{y}}=$ $r_{0} \cos \theta_{0} \hat{\mathbf{x}}+r_{0} \sin \theta_{0} \hat{\mathbf{y}}$ and $\mathbf{r} \equiv x \hat{\mathbf{x}}+y \hat{\mathbf{y}}=r \cos \theta \hat{\mathbf{x}}+$ $r \sin \theta \hat{\mathbf{y}}$, respectively.

Following [11], a generic HB parameterized by the "twist-angle" $\alpha$ is synthesized via coherent superposition of GBs launched from a circular aperture of radius $R_{0}$ at the waist plane $(z=0)$, with optical axes directed along the unit vector

$$
\begin{aligned}
\mathbf{u}_{\alpha} & =\left(r_{0} / L\right)\left[\cos \theta_{0}-\cos \left(\theta_{0}-\alpha\right)\right] \hat{\mathbf{x}} \\
& +\left(r_{0} / L\right)\left[\sin \theta_{0}-\sin \left(\theta_{0}-\alpha\right)\right] \hat{\mathbf{y}}+\hat{\mathbf{z}}
\end{aligned}
$$

As shown in Fig. 2a, these optical axes are the generators of hyperboloids. Varying the twist-angle $\alpha$ allows continuous spanning from the FM configuration ( $\alpha=0$, cylindrical degenerate, cf. Fig. $2 \mathrm{~b})$ to the CM configuration ( $\alpha=\pi$, conical degenerate, cf. Fig. 2c). The GB spot size at the waist is chosen according to the minimum-spreading criterion,

$$
w_{0}=\sqrt{\frac{L}{k_{0}}},
$$

where $k_{0}=2 \pi / \lambda_{0}$ denotes the free-space wavenumber (with $\lambda_{0}$ denoting the free-space wavelength). This corresponds to locating the mirror planes exactly at the Rayleigh distance [12], i.e., $z_{R} \equiv$ $k_{0} w_{0}^{2} / 2=L / 2$. For this HB family, the wavefronts at the mirror location are roughly approximated by the "fiducial" spheroids [11]

$$
z=S_{\alpha}(r) \equiv \sqrt{\left(\frac{L}{2}\right)^{2}-r^{2} \sin ^{2}\left(\frac{\alpha}{2}\right)},
$$

which degenerate into planar and spherical surfaces in the FM $(\alpha=0)$ and $\mathrm{CM}(\alpha=\pi)$ case, respectively. It is well-known from the theory of gradedphase mirrors [13] that, in order for the optical cavity to support the desired HB profile $U_{\alpha}$ as the fundamental eigenmode, the mirrors need to be shaped according to the fiducial spheroid in (3) with a correction

$$
h_{\alpha}(r)=\frac{\arg \left[U_{\alpha}\left(r, S_{\alpha}\right)\right]-\arg \left[U_{\alpha}\left(0, S_{\alpha}\right)\right]}{k_{0}},
$$

so as to match the HB wavefront. For the FM $(\alpha=0)$ and $\mathrm{CM}(\alpha=\pi)$ cases, this reduces to the Mexican-hat-shaped profile in [6].

In [11], the following symmetry relations were derived (with $\Lambda$ denoting a complex constant)

$$
\begin{gathered}
U_{-\alpha}\left(r, S_{\alpha}\right)=U_{\alpha}\left(r, S_{\alpha}\right), \\
\frac{U_{\pi-\alpha}\left(r, S_{\alpha}\right)}{\Lambda}=\frac{U_{\alpha}^{*}\left(r, S_{\alpha}\right)}{\Lambda^{*}},
\end{gathered}
$$
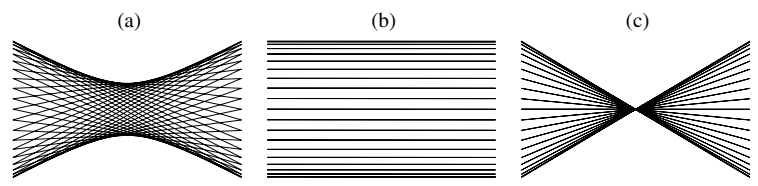

Figure 2: HB geometrical construction (see text). (a) Generic HB $(0 \leq \alpha \leq \pi)$; (b) FM beam $(\alpha=0)$;

(c) CM beam $(\alpha=\pi)$.

$$
h_{\pi-\alpha}(r)=-h_{\alpha}(r),
$$

which generalize in part the FM-CM duality relations in $[9,10]$.

\subsection{Analytic Structure and Properties}

In [14], generalizing certain results in [3], we derived a GL beam expansion for a generic $\mathrm{HB}$

$$
U_{\alpha}(r, z)=\sum_{m=0}^{\infty} A_{m}^{(\alpha)} \Psi_{m}(r, z),
$$

where $\Psi_{m}(r, z)$ denote the standard GL beam propagators [12]

$$
\begin{aligned}
\Psi_{m}(r, z) & =\frac{w_{0}}{w(z)} \psi_{m}\left[\frac{\sqrt{2} r}{w(z)}\right] \exp \left[i \frac{k_{0} r^{2}}{2 R(z)}\right] \\
& \times \exp \left\{i\left[k_{0} z-(2 m+1) \Phi(z)\right]\right\}
\end{aligned}
$$

and the expansion coefficients $A_{m}^{(\alpha)}$ are given by

$$
A_{m}^{(\alpha)}=(-\cos \alpha)^{m} \frac{\sqrt{2} w_{0}^{2}}{R_{0}^{2}} P\left(m+1, \frac{R_{0}^{2}}{2 w_{0}^{2}}\right) .
$$

In (7) and (8),

$$
\psi_{m}(\xi)=\sqrt{2} \exp \left(-\frac{\xi^{2}}{2}\right) L_{m}\left(\xi^{2}\right)
$$

are orthonormal GL basis functions, with $L_{n}(\zeta)$ denoting an $n$ th-order Laguerre polynomial [15, Chap. 22],

$$
\begin{array}{r}
w(z)=w_{0} \sqrt{1+\left(\frac{z}{z_{R}}\right)^{2}}, R(z)=z+\frac{z_{R}^{2}}{z}, \\
\Phi(z)=\arctan \left(\frac{z}{z_{R}}\right)
\end{array}
$$

denote the standard GB spot size, wavefront radius of curvature, and Gouy phase, respectively [12], and $P(n, \xi)$ denotes an incomplete Gamma function $[15$, Eq. (6.5.13)].

Figure 2 illustrates some representative results for the HB intensity distribution on the fiducial 


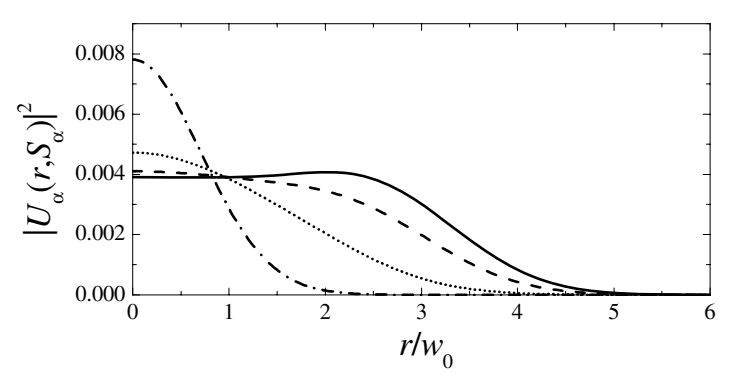

Figure 3: HB field distribution evaluated on the fiducial surface $z=S_{\alpha}(r)$, for different values of the twist-angle parameter $\alpha$. Parameters: $L=4 \mathrm{~km}$, $\lambda_{0}=1064 \mathrm{~nm}, w_{0}=\sqrt{L \lambda_{0} /(2 \pi)}=2.603 \mathrm{~cm}$, and $R_{0}=4 w_{0}=10.4 \mathrm{~cm}$. Continuous curve: $\alpha=0 ;$ dashed: $\alpha=0.1 \pi, 0.9 \pi ;$ dotted: $\alpha=$ $0.2 \pi, 0.8 \pi$; dotted-dashed: $\alpha=0.5 \pi$.

surface, for a parametric configuration of potential interest for Advanced-LIGO (as in [6, 11]) and various values of the twist-angle $\alpha$, illustrating the gradual transition from the Gaussian $(\alpha=\pi / 2)$ to the mesa $(\alpha=0, \pi)$ profile. In all examples, the GL series in (6) was truncated according to the criterion $\left|A_{M}^{(\alpha)}\right|<10^{-3}\left|A_{0}^{(\alpha)}\right|$, with $M$ denoting the index of the last retained term. It was verified, by numerical comparison with the integral representation in [11], that this ensures a relative error in the intensity $\lesssim 0.1 \%$ and an absolute error in the mirror correction (cf. (4)) $\lesssim 10^{-4} \lambda_{0}$, over the significantly illuminated portion of the mirror.

The analytic GL expansion in (6) sets the stage for a generalization, to arbitrary twist-angles, of the duality relations in $[9,10]$. In this framework, we consider a class of $\sigma$-parameterized ${ }^{1}$ generalized Hankel transform (HT) operators defined as

$$
\begin{aligned}
\mathcal{H}_{w_{0}}^{(\sigma)}[F(r)] & \equiv \frac{4}{w_{0}^{2}(1+\sigma)} \int_{0}^{\infty} r_{0} d r_{0} F\left(r_{0}\right) \\
& \times \exp \left[-\frac{\left(r^{2}+r_{0}^{2}\right)(1-\sigma)}{w_{0}^{2}(1+\sigma)}\right] \\
& \times J_{0}\left[\frac{4 r r_{0} \sqrt{\sigma}}{w_{0}^{2}(1+\sigma)}\right], \quad \sigma \geq-1 .
\end{aligned}
$$

The generalized HT operator in (11) can be shown (see [16, p. 43] and [14] for more details) to admit as eigenfunctions the GL basis functions in (9),

$$
\mathcal{H}_{w_{0}}^{(\sigma)}\left[\psi_{m}\left(\frac{\sqrt{2} r}{w_{0}}\right)\right]=(-\sigma)^{m} \psi_{m}\left(\frac{\sqrt{2} r}{w_{0}}\right) .
$$

${ }^{1}$ For $\sigma<-1$, the integral in (11) diverges for the beams of interest here (decaying as $O\left[\exp \left(-r^{2} / w_{0}^{2}\right)\right]$ in the waist plane), and the following definition should be used: $\mathcal{H}_{w_{0}}^{(\sigma)}[F(r)]=\mathcal{H}_{w_{0}}^{(-\sigma)}\left\{\mathcal{H}_{w_{0}}^{(1)}[F(r)]\right\}$.
Application of the generalized HT (11) to the GL expansion in (6) reveals, via (12), the functional relation between the field distributions of two generic $\alpha_{1,2}$-parameterized HBs at the waist plane $(z=0)$ and at the fiducial spheroid $\left(z=S_{\alpha_{1,2}}\right)$, viz.,

$$
\begin{gathered}
U_{\alpha_{2}}(r, 0) \stackrel{\mathcal{H}_{w_{0}}^{(\sigma)}}{\longleftrightarrow} U_{\alpha_{1}}(r, 0), \\
\frac{U_{\alpha_{2}}\left(r, S_{\alpha_{2}}\right)}{\exp \left(i \frac{k_{0} r^{2} \cos \alpha_{2}}{2 L}\right)} \stackrel{\mathcal{H}_{\sqrt{2} w_{0}}^{(\sigma)}}{\longleftrightarrow} \frac{U_{\alpha_{1}}\left(r, S_{\alpha_{1}}\right)}{\exp \left(i \frac{k_{0} r^{2} \cos \alpha_{1}}{2 L}\right)}, \\
\sigma=-\frac{\cos \alpha_{2}}{\cos \alpha_{1}} .
\end{gathered}
$$

The generalized HT in (13) extends the symmetry relations in (5) to the most general case, and admits a suggestive analytic interpretation in terms of a fractional Fourier operator of complex order [17]-[19]. These operators admit a physical interpretation in terms of propagation through a paraxial optical system described by a complex ABCD matrix, which, for the HT in (11), is found to be

$$
\begin{aligned}
{\left[\begin{array}{ll}
A & B \\
C & D
\end{array}\right] } & =\left[\begin{array}{cc}
i \frac{(1-\sigma)}{2 \sqrt{\sigma}} & \frac{k_{0} w_{0}^{2}(1+\sigma)}{4 \sqrt{\sigma}} \\
-\frac{(1+\sigma)}{k_{0} w_{0}^{2} \sqrt{\sigma}} & i \frac{(1-\sigma)}{2 \sqrt{\sigma}}
\end{array}\right] \\
& =\left[\begin{array}{cc}
\cos \left(\frac{\pi \gamma}{2}\right) & \frac{L}{2} \sin \left(\frac{\pi \gamma}{2}\right) \\
-\frac{2}{L} \sin \left(\frac{\pi \gamma}{2}\right) & \cos \left(\frac{\pi \gamma}{2}\right)
\end{array}\right] .
\end{aligned}
$$

In $(14 \mathrm{~b})$, the parameter $\gamma$ denotes the complex order of the transform, defined as [19]

$$
\begin{aligned}
\gamma & \equiv-i \frac{2}{\pi} \log \left[A \sqrt{\frac{D}{A}}+i B \sqrt{\frac{-C}{B}}\right] \\
& =1+i \frac{\log (\sigma)}{\pi} .
\end{aligned}
$$

Alternative optical interpretations in terms of propagation through complex Gaussian ducts, as well as through optical systems composed of self-imaging components and Gaussian apertures, can be found in [12], [17]-[19]. Figure 4 illustrates schematically the range of admissible values for the complex order $\gamma$ in (15), over the meaningful $\left(\alpha_{1}, \alpha_{2}\right)$-range: The real part can either be 1 or 0 , while the imaginary part is generally nonzero, except along the two diagonals $\alpha_{1}=\alpha_{2}$ and $\alpha_{1}=\pi-\alpha_{2}$ where the generalized HT operator in (11) reduces to the identity and ordinary HT operator, respectively.

\section{CONCLUSIONS}

In this paper, we have presented a study of the analytic structure and properties of a family of 

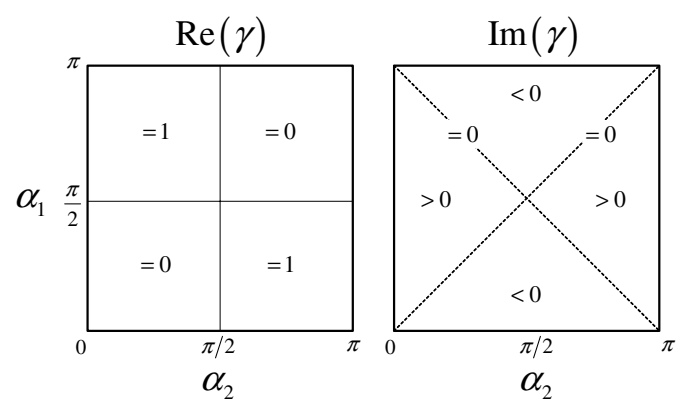

Figure 4: Admissible values of the complex order $\gamma$ (15) of the generalized HT operator in (11) relating the field distributions of two BT hyperboloidal beams with generic twist-angles, $\alpha_{1}$ and $\alpha_{2}$ (see text).

HBs of interest for thermal-noise reduction in advanced gravitational wave interferometric detectors. Specifically, we have introduced an analytic GL-type representation for generic HBs, and have derived a complex-Fourier-transform-based generalization of the duality relations in $[9,10]$ for the dominant eigenmode.

Current and future investigations are aimed at extending the above results, to higher-order (axisymmetric and multipolar) modes, and addressing the optimization of the optical cavities of gravitational wave interferometric detectors in terms the best tradeoff between thermal-noise and tiltinstability reduction.

\section{References}

[1] S. De Silvestri, P. Laporta, V. Magni, and O. Svelto, "Solid-state laser unstable resonators with tapered reflectivity mirrors: The superGaussian approach," J. Quantum Electron., vol. 24, No. 6, pp. 1172-1177, 1988.

[2] F. Gori, "Flattened Gaussian beams," Opt. Commun., vol. 107, No. 5-6, pp. 335-341, 1994.

[3] C. J. R. Sheppard and S. Saghafi, "Flattened light beams," Opt. Commun., vol. 132, No. 12, pp. 144-151, 1996.

[4] A. A. Tovar, "Propagation of flat-topped multi-Gaussian laser beams," J. Opt. Soc. Am. $A$, vol. 18, No. 8, pp. 1897-1904, 2001.

[5] Y. Li, "Light beams with flat-topped profiles," Opt. Lett., vol. 27, No. 12, pp. 1007-1009, 2002.

[6] E. D'Ambrosio, "Nonspherical mirrors to reduce thermoelastic noise in advanced gravi- tational wave interferometers," Phys. Rev. D, vol. 67, No. 10, 102004, 2003.

[7] http://www.ligo.caltech.edu/advLIGO

[8] J. Agresti, E. D’Ambrosio, R. DeSalvo, J. Mackowski, A. Remillieux, B. Simoni, M. Tarallo, and P. Willems, "Flat top beam profile cavity prototype," in Laser Beam Shaping VI, Proc. SPIE, vol. 5876, SPIE, Bellingham, WA, USA, 2005.

[9] P. Savov and S. Vyatchanin, "Estimate of tilt instability of mesa-beam and Gaussian-beam modes for advanced LIGO," Phys. Rev. D, vol. 74, No. 8, 082002, 2006.

[10] P. Savov, J. Agresti, Y. Chen, E. D’Ambrosio, "A duality relation between non-spherical optical cavities and its application to gravitational-wave detectors," preprint ArXiv:gr-qc/0511062, 2005.

[11] M. Bondarescu and K. S. Thorne, "New family of light beams and mirror shapes for future LIGO interferometers," Phys. Rev. D, vol. 74, No. 8, 082003, 2006.

[12] A. E. Siegman, Lasers, University Science Books, Mill Valley, CA, 1986.

[13] C. Paré and P. A. Bélanger, "Custom laser resonators using graded-phase mirrors: Circular geometry," IEEE J. Quantum Electron., vol. 30, No. 4, pp. 1141-1148, 1994.

[14] V. Galdi, G. Castaldi, V. Pierro, I.M. Pinto, J. Agresti, E. D'Ambrosio, and R. DeSalvo, "Analytic structure of a family of hyperboloidal beams of potential interest for advanced LIGO," Phys. Rev. D, vol. 73, No. 12, 127101, 2006.

[15] M. Abramowitz and I. A. Stegun, Handbook of Mathematical Functions, Dover, New York, NY, 1964

[16] A. Erdélyi, M. F. Oberhettinger, and F. G. Tricomi, Tables of Integral Transforms, Vol. 2, McGraw-Hill, New York, NY, 1954.

[17] C.-C. Shih, "Optical interpretation of a complex-order Fourier transform," Opt. Lett., vol. 20, No. 10, pp. 1178-1180, 1995.

[18] L. M. Bernardo and O. D. D. Soares, "Optical fractional Fourier transform with complex order," Appl. Opt., vol. 35, No. 17, pp. 31633166, 1996.

[19] C. Wang and B. Lü, "Complex-order Fourier transforms and their implementation," Optik, vol. 113, No. 8, pp. 337-342, 2002. 